\title{
A comparison of the effectiveness of intubation using a McGrath Series 5 videolaryngoscope with either a Truflex articulating stylet or a standard intubation stylet in a group of medical students
}

BY MARCIN CIERNIAK, MARCIN NOWAKOWSKI, DARIUSZ TIMLER, TOMASZ GASZYŃSKI

\section{Abstract}

Background. Intubation is the optimal method for opening the airway and effective ventilation of the patient. However, there are occasional problems with intubation, especially in patients with injuries under pre-hospital conditions. Therefore, it is important to identify devices, e.g., videolaryngoscopes or guides, which may facilitate and shorten the procedure. This study addresses the use of a Truflex articulated guide with a videolaryngoscope.

Objectives. The main objective of the study is to evaluate the effectiveness of intubation using a Truflex articulating stylet with a McGrath videolaryngoscope, and to determine whether the average time of intubation using a Truflex articulating stylet is shorter than that using a standard intubation stylet.

Materials and methods. The study involved 43 full-time 5 th year medical students. All tests were performed on training manikins with a difficult airway in January 2013. Chi-square test was used for statistical analysis with a significance level of $p$ 
$<$ o.01. Calculations were performed using the Statistica package.

Results. Intubation using a McGrath videolaryngoscope with a Truflex articulating stylet was more effective than that using a standard intubation stylet with the same laryngoscope: $71 \%$ as compared to $40 \%$. The mean time of successful intubation using a Truflex articulating stylet was shorter than that using a standard intubation stylet guide (31.1 $\pm 12.8 \mathrm{~s}$ and $39.8 \pm 12.4 \mathrm{~s}$, respectively).

Conclusions. The mean time of tracheal intubation using a Truflex articulating stylet is shorter than that using a standard intubation stylet. Intubation is also more effective when a Truflex articulating stylet is used together with a McGrath videolaryngoscope.

Key words: Truflex articulating stylet, Truflex, intubation, McGrath

\section{Introduction}

Tracheal intubation is regarded as the optimal means of securing the airway. It provides effective ventilation for patients and protects them from regurgitation. $(1,2)$ However, even when performed by an experienced anesthesiologist, intubation might not be a simple procedure, and there are complications known as difficult intubation or intubation of a difficult airway. Nevertheless, difficult intubations may be foreseen. According to ASA (American Society of Anesthesiologists Physical Status Classification System) standards, a difficult intubation occurs in the event of deviations from normal anatomy or under pathological conditions. Of course, it is not only anatomical conditions that may be responsible for difficult intubations: they are often encountered under prehospital conditions, e.g., if serious damage has occurred to the craniofacial area, or in the presence of swelling, heavy bleeding, or foreign bodies. Difficult intubation is not only a problem faced by the paramedic service; it represents one of the main causes of death and morbidity associated with anesthesia. (3) In cases of difficult intubation, there is a real risk that the patient may not be intubated or, more importantly, ventilated. It is therefore important to find ways to both facilitate and accelerate the process of intubation using equipment such as videolaryngoscopes and guides.

The present study examined the use of a Truflex articulating stylet (Truphatek, Israel), which has an articulated tip, with a McGrath videolaryngoscope (Aircraft 
Medical Limited, Edinburgh United Kingdom). According to the manufacturers, both of these devices facilitate shorter intubation times. The flexion range of the Truflex tip $\left(30^{\circ}\right.$ to $\left.60^{\circ}\right)$ gives it an advantage over the standard guide. The main aim of this study was to evaluate the effectiveness of intubation using a combination of the new guide and a McGrath videolaryngoscope. Another objective was to determine whether the mean intubation time is shorter using a Truflex articulating stylet than a standard intubation stylet.

\section{Materials and methods}

The present study involved 43 full-time medical students in their 5 th year of university. The students were recruited for the study after completing a course which dealt with instrumented opening of the airway under pre-hospital conditions. Tests were conducted in the teaching rooms of the Medical University. All data were obtained in January 2013.

Prior to testing, each participant was first given several minutes to familiarize themselves with a McGrath Series 5 videolaryngoscope, which was used in combination with either a Truflex articulating stylet (figure 1) or a standard rigid intubation stylet to perform direct laryngoscopy. Most importantly, prior to tests, a demonstration was given on medical manikins with a difficult airway, showing correct intubation using both guides and a laryngoscope.

Measurement began the moment the student reached for the intubation equipment, which had been previously prepared and laid out: a laryngoscope, intubation tubes, syringes for filling the balloon, as well as the aforementioned stylets. Each trainee had an assistant in the form of another student to best replicate the intubation conditions of working on a patient in a pre-hospital setting at the site of an accident. The measurement ended at the point when the position of the endotracheal tube in the trachea was ascertained by listening to the upper abdomen and lung fields.

The maximum time allowed for the test was $60 \mathrm{~s}$, this being twice as long as the recommended time allowed for intubation. Each test was performed three times for each operator. If none of the three attempts were successful, a fourth attempt was permitted.

Chi-square test was used for statistical analysis with $\mathrm{p}<0.01$ being the limit of 
statistical significance. All calculations were performed using Statistica.

\section{Results}

The distributions for both guides were right skewed (positive skewness = 0.2; o.09), which may be due to the fact that in the case of failed attempts the intubation times often exceeded half of the time allocated to the test (30 seconds). In both cases, the distributions were also platykurtic (negative kurtosis = -1.22; -0.34) due to an excess of extreme results: the majority of participants either intubated relatively quickly or had to use the full 6o seconds. The effectiveness of intubation with the use of a McGrath Series 5 videolaryngoscope was higher when using a Truflex articulating stylet than a standard guide: $71 \%$ (93 successful attempts out of 130) as opposed to 40\% (59 successful attempts out of 144 (figure 2) (Chi-square test $=6.31$ for critical values -5.412 to 5.412 , with the level of statistical significance being $\mathrm{p}<0.01$ ). When using a standard guide, $42 \%$ of the male students performed a successful intubation as compared to $37 \%$ of the female students; however, in the case of the Truflex articulating stylet, the female students were found to be more efficient than the male students ( $78 \%$ vs. $65 \%$ effectiveness, respectively). There is no statistically significant association between the effectiveness of intubation and the sex of the participants. Chisquare test for standard stylet $\mathrm{p}=5,8$ and for Truflex stylet $\mathrm{p}=1$.o. The mean time of successful intubations performed with a Truflex was shorter than that for the traditional guide: $31.1 \pm 12.8 \mathrm{~s}$ as compared to $39.8 \pm 12.4 \mathrm{~S}$ (table 1 ). Half of the intubations were performed in less than $37 \mathrm{~s}$ with a standard intubation stylet, and half were performed in less than $34 \mathrm{~s}$ with a Truflex.

In some tests, after three failed attempts, it was necessary to add a fourth attempt; this situation occurred in 15 cases for the traditional guide but only once for the Truflex articulating stylet. The shortest time for successful intubation was $18 \mathrm{~s}$ with a rigid intubation stylet, but only $11.5 \mathrm{~s}$ with a Truflex.

\section{Discussion}

Occlusion of the upper airway represents a serious threat to life and requires immediate opening. While the optimal method remains intubation, the frequency of occurrence of difficulties during intubation carried out through direct 
laryngoscopy is as high as 10\%-13\%. (4) Intubation may be particularly difficult under pre-hospital conditions, especially where impacts to the craniofacial area are complicated by heavy bleeding or swelling. Thus, it is important to take advantage of tools such as guides and videolaryngoscopes, which allow better visualization of the entry to the larynx.

This study demonstrated that intubation performed with a Truflex articulating stylet and a McGrath videolaryngoscope by a group of students who had little experience with the equipment was not only more effective than intubation performed using a traditional guide, but also the mean intubation time was shorter. It may therefore be assumed that a combined use of these devices reduces the time of intubation even if it is carried out by users without extensive clinical experience. Similar observations were made by Shirgoski and Bell. $(5,6)$ Bell (7) reported that intubation procedures carried out using Truflex articulating stylets resulted in less laryngoscope movement and caused less damage to the teeth or the inside of the oral cavity; hence, less damage is likely to be done during the intubation process.

Tests with the Truflex articulating stylet show that its use can reduce the time needed for intubation of patients with injuries. Al-Qasmi (8) noted the same in his study protocol. Furthermore, for an even faster intubation, it is worth considering the additional use of a McGrath videolaryngoscope. In this context, Taylor noted greater intubation efficiency for this videolaryngoscope than for the Macintosh laryngoscope. $(9,10)$ The efficiency observed in the present study $(71 \%)$ is slightly less than that found by Ray, who performed a very similar study on a group of medical students: the effectiveness of the use of a McGrath videolaryngoscope was reported to be as high as $85 \%$ without a guide. (11) The present study, similarly to other studies on the use of videolaryngoscopy in difficult intubations performed on medical phantoms by medical students with little experience, $(7,12-14)$ shows that videolaryngoscopes are great tools for teaching intubation to future doctors. The above-mentioned studies differ with respect to the difficulty of laryngoscopy, the number of intubation attempts, and their effectiveness; however, all indicate that greater effectiveness was obtained by the use of videolaryngoscopes. In the present study, the mean time for successful intubation using a videolaryngoscope combined with a Truflex articulating stylet (31.1 $\pm 12.8 \mathrm{~s})$ was shorter than that when a standard intubation stylet was used. These results confirm those of a German study by Reus, in which the mean intubation time with a Truflex articulating stylet was found to be $26.4 \pm 5.5 \mathrm{~s}$. Similarly to Bell, Reus recruited a 
group of anesthesiologists, but in this case the subjects had completed less than one year of professional internship; moreover, a McGrath videolaryngoscope was not used. (15-17) When the benefits of the combined use of a reusable Truflex articulating stylet and a McGrath videolaryngoscope are taken into consideration, it can be seen that these devices are to be recommended for difficult intubation cases.

\section{Limitations}

The study was limited to several groups of medical students and was conducted only at one university. The study group consisted of 43 members and was not large enough to generalize the results to all medical students; this was caused by the limited number of classes with students. Student's t-test for independent groups was used.

\section{Conclusions}

The present study shows that the mean time of tracheal intubation with the use of a reusable Truflex articulating stylet and a McGrath videolaryngoscope is shorter than that when a standard intubation stylet is used.

In the presented experiments, a greater percentage of successful first-time intubation attempts was noted when a Truflex articulating stylet was used.

Also in the present study, intubation of the trachea was performed in a precise way more often when a Truflex articulating stylet was used.

Table 1. List of successful intubation attempts and the average duration of the tests. Numbers in columns signify $\mathrm{M} \pm \mathrm{SD}$ and $\mathrm{N}$.

\begin{tabular}{lll} 
& $\begin{array}{l}\text { Rigid standardintubation stylet } \\
\text { guide }\end{array}$ & $\begin{array}{l}\text { Truflex articulating } \\
\text { stylet }\end{array}$ \\
\hline Total number of attempts & $59 / 144$ & $93 / 130$ \\
\hline Mean time of a successful attempt & $39.8 \pm 12.4 \mathrm{~S}$ & $31.1 \pm 12.8 \mathrm{~s}$ \\
\hline Mean time of a first successful attempt & $62.8 \pm 27 \mathrm{~s}$. & $47.8 \pm 33.7 \mathrm{~S}$. \\
\hline Mean time of a second successful & $58.5 \pm 33 \mathrm{~S}$. & $41 \pm 20.6 \mathrm{~S}$.
\end{tabular}


attempt

Mean time of a third successful attempt $43 \pm 20.5 \mathrm{~S}$.

$36.5 \pm 20.7 \mathrm{~s}$.

Figure 2. The effectiveness of intubation.

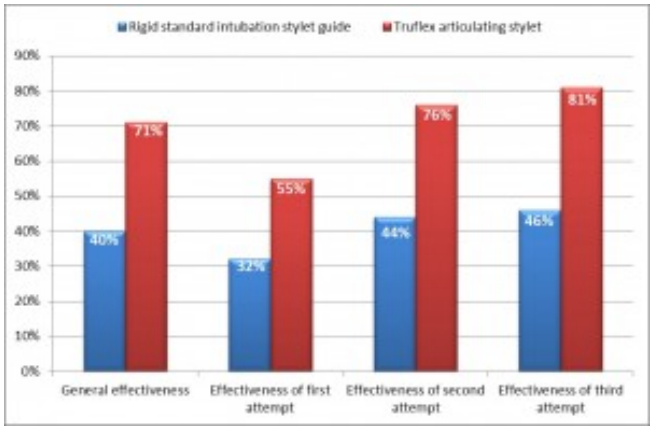

Figure 1. Truflex articulating stylet (source: manufacturer marketing materials).

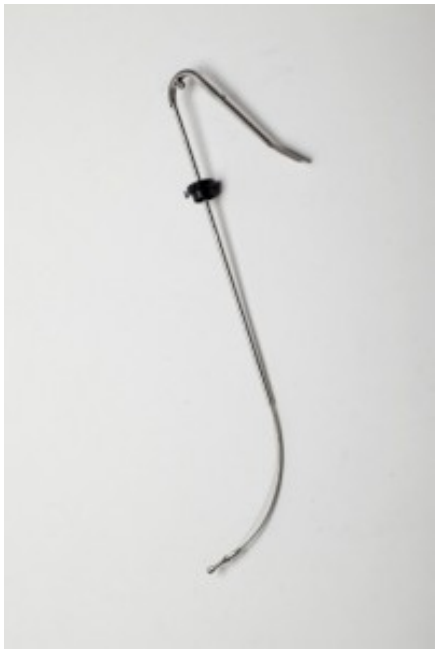

\section{References}

1. JakubiakJ, Gaszynski T, Gaszynski W. Nadkrtaniowe urządzenia do udrożnienia górnych dróg oddechowych z możliwością przeprowadzenia intubacji dotchawiczej - porównanie Cobra PLA i ILMA. Anaesth Resusc Med 2009;3:31-6.

2. Eftekharian Jahromi HR. Guide Wire J-Tip Technique for Easy Exchange of In Situ Endotracheal Tubes. Iran J Med Sci 2012;37:141-3.

3. Kluger MT, Tham EJ, Coleman NA, Runciman WB, Bullock FM. Inadequate preoperative evaluation and preparation: a review of 197 reports from the Australian Incident Monitoring Study. Anaesthesia 2000;55:1173-8.

4. Ekweghariri C, Grunewald G, Kramcha T, Rahman MA, Atlas G. V-Scope 
Extendable Intubation Stylet. 38th Annual Northeast Bioengineering Conference (NEBEC) 16-18 March 2012. Philadelphia; 2012. p. 61-2. DOI:

10.1109/NEBC.2012.6206962

5. Bell SF, Davies N, Crossley A. Glidescope guided intubation: Comparison of Frova introducer, GlideRite Rigid, GlideRite Auto and Truphatek Truflex stylets in a simulated difficult intubation: 19AP5-2. Eur J Anaesthesiol 2011;28:235.

6. Shirgoska B, Netkovski J. New techniques and devices for difficult airway management. Acta Clin Croat 2012;51:457-61.

7. Hirabayashi Y. Airway scope versus Macintosh laryngoscope: a manikin study. Emerg Med J 2007;24:357-8.

8. Al-Qasmi A, Al-Alawi W, Malik AM, Khan RM, Kaul N. Trials. Assessment of Truflex articulating stylet versus conventional rigid Portex stylet as an intubation guide with the D-blade of $\mathrm{C}$-Mac videolaryngoscope during elective tracheal intubation: study protocol for a randomized controlled trial. BioMed Central.2013;14:298.

9. Taylor AM, Peck M, Launcelott S, Hung OR, Law JA, MacQuarrie K, et al. The McGrath ${ }^{\circledR}$ Series 5 videolaryngoscope vs the Macintosh laryngoscope: a randomised, controlled trial in patients with a simulated difficult airway. Anaesthesia 2013;68:142-7.

10. Wetsch WA, Spelten O, Hellmich M, Garlitscheck M, Padosch SA, Lier H, et al. Comparison of different video laryngoscopes for emergency intubation in a standardized airway manikin with immobilized cervical spine by experienced anaesthetists. Resuscitation 2012;83:740-5.

11. Ray DC, Billington C, Kearns PK, Kirkbride R, Mackintosh K, Reeve CS, et al. A comparison of McGrath and Macintosh laryngoscopes in novice users: a manikin study. Anaesthesia 2009;64:1207-10.

12. Hirabayashi Y, Seo N. Tracheal intubation by non-anaesthetist physicians using the Airway Scope. Emerg Med J 2007;24:572-3.

13. Miki T, Inagawa G, Kikuchi T, Koyama Y, Goto T. Evaluation of the Airway Scope, a new video laryngoscope, in tracheal intubation by naive operators: a manikin study. Acta Anaesthesiol Scand 2007;51:1378-81.

14. Tan BH, Liu EHC, Lim RTC, Liow LMH, Goy RWL. Ease of intubation with the Glidescope or Airway Scope by novice operators in simulated easy and difficult airways - a manikin study. Anaesthesia 2009;64:187-90.

15. Reus E, Liening K, Wrobel M, Grundmann U. Flexible fiberoptic versus Parker Flex-It, Truflex and hockey stick formed stylet as an intubation guide with the videolaryngoscope McGRATH Series 5 in a simulated airway: 19AP5-10. Eur J 
Anaesthesiol 2012;29:241.

16. Reus E, Liening K, Wrobel M, Grundmann U. Evaluation of the success rate of the intubation with the videolaryngoscope McGrath ${ }^{\circledR}$ in combination with the direction giving Truflex TM stylet versus a hockeystick formed stylet in a simulated airway: 19AP5-8. Eur J Anaesthesiol 2011; 28:236-7.

17. Reus E, Grundmann U, Liening K, Wrobel M. Parker Flex-It intubation stylet versus a 90-degree curved stylet during intubation with the McGrath videolaryngoscope performed by novices: a manikin study with 5 airway scenarios. J Clin Anesth 2013;25:624-8.

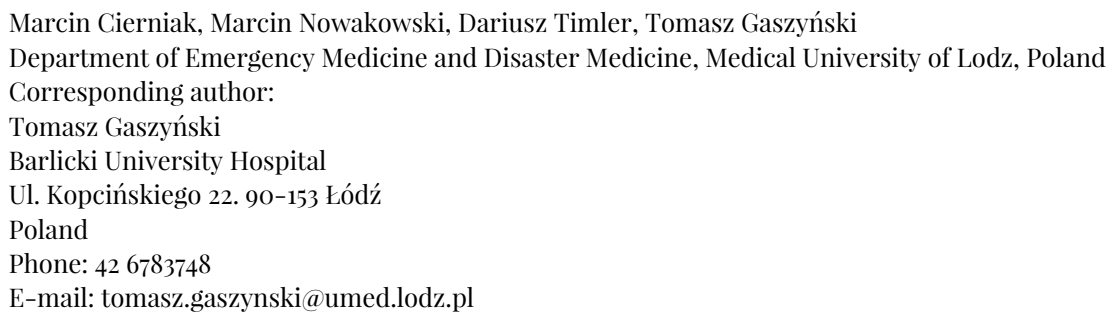

